\section{Best in show but not best weight: using photographs to determine the weight status of show dogs}

\section{Zackary R Such, Alexander J German}

University of Liverpool, Neston, Cheshire, UK

Overweight dogs are prevalent in the pet population, but owners may fail to recognise it. Previous studies suggest that owners often wrongly perceive overweight dogs to be in normal condition. The body shape of dogs attending shows might influence owners' perceptions of body shape, with online images of an overweight show winner having a particularly damaging effect. The aim of the current study was to review internet images of dogs, which placed highly at a national UK dog show, to determine breed-specific prevalence of overweight.

The study protocol was approved by the University of Liverpool Research Ethics Committee. First, 28 breeds were selected, 14 of which were obese-prone, and 14 were not. Breeds were chosen from all Kennel Club categories, and non-obese-prone breeds were matched, for shape and size, to the obese-prone breeds. Using an online search engine (Google Images; http://www.google.com/imghp), one operator then identified 40 images of dogs per breed, all of which had appeared at a major national UK show (Crufts). For each photograph, information was collated regarding breed, breed group, year of competition, sex, coat colour, and placing in show. The data were coded and recorded in a computer spreadsheet (Excel, Microsoft), and each photograph assigned an anonymous code. A second observer then independently viewed the coded photographs, and scored body condition using a validated photographic scoring method. Photographs were assessed in a single sitting, and scores recorded in a second spreadsheet, identified by the code only. Afterwards, the images were deleted, and data from both spreadsheets were combined.

A total of 1120 photographs of dogs were obtained, of which 960 proved suitable for assessing body condition, with all unsuitable images being from longhaired breeds. Of the 960 dogs, 0 (0\%) were underweight, 708 were in ideal condition (74\%) and 252 (26\%) were overweight. Conditional logistic regression demonstrated that pugs (odds ratio $[O R]=10.7$, $\mathrm{P}<0.001)$, basset hounds $(\mathrm{OR}=5.6, \mathrm{P}<0.001)$ and Labrador retrievers $(O R=4.5, P<0.001)$ were most likely to be overweight, whilst standard poodles $(\mathrm{OR}=0.1, \mathrm{P}=0.01)$, Rhodesian ridgebacks $(O R=0.2, P=0.01)$, Hungarian vizslas $(O R=0.2$, $P=0.01)$ and Doberman pinschers $(O R=0.2, P=0.01)$ were least likely to be overweight.

Given the proportion of show dogs from some breeds that are overweight, breed standards should be redefined to be consistent with a dog in optimal body condition.

\section{Transitioning of porcine insulin lente-treated diabetic cats to a human-recombinant protamine zinc preparation}

\section{Ruth Gostelow', David Church', Stijn Niessen ${ }^{1,2}$}

The Royal Veterinary College, Hatfield, UK
Newcastle Medical School, Newcastle, UK

\section{BACKGROUND}

Feline diabetes mellitus (DM) is currently recommended to be treated through addressing underlying disease processes, twice daily insulin injections and a low carbohydrate diet. Good glycaemic control is one of the few factors that have been associated with diabetic remission, though a proportion of cats fail to achieve this on porcine insulin lente (Caninsulin ${ }^{\circledR}$, MSD Animal Health), previously the only veterinary-licensed insulin for use in diabetic pets in Europe.

\section{AIM}

This pilot study evaluated a protocol for transitioning Caninsulin-treated diabetic cats onto newly licensed human-recombinant protamine zinc insulin (ProZinc ${ }^{\circledR}$, Boehringer Ingetheim).

\section{METHODS}

Recently ( $<5$ months) diagnosed diabetic cats, treated with Caninsulin BID for at least 6 weeks, were recruited. A full history, physical examination, diabetic clinical score (DCS; range 0 [no diabetic signs] -12 [many diabetic signs]), fructosamine concentration and 24-hour blood glucose-curve (BGC) were performed before and 1 month after the transition to ProZinc (start dose: 0.2-0.7 units/kg BID), following a set protocol of weekly BGCs and dose adjustments (0.5-1 unit change/injection/week guided by nadir). Cats were excluded if screening (biochemistry, urinalysis, fPLI, TT4, IGF-1, abdominal ultrasound) identified: ketoacidosis, clinical pancreatitis, glucocorticoid/progestogen administration, hyperthyroidism, acromegaly or other conditions impairing treatment response or compliance with the trial's protocol. Changes were assessed using Wilcoxon Signed Rank test $(p<0.05)$.

\section{RESULTS}

Fourteen cats were recruited (10 MN, 4 FN; median [range] age: 10 years [6.0-15.4]); cats received a median (range) of 0.5 unit/kg (0.2-1.2) Caninsulin BID and had a median (range) DCS of 2 (0-9). Median (range) BGC value at time of Caninsulin treatment was $11.5 \mathrm{mmol} / \mathrm{l}(8.8-22.2)$ and median fructosamine (range) $445 \mu \mathrm{mol} / \mathrm{l}$ (270-619). One month after transitioning to Prozinc cats were receiving a median of 0.5 unit/kg (0.1-0.8) BID and had a significantly lower median DCS of 1 (range $0-7)$; median $B G C$ value $(7.5 \mathrm{mmo} / \mathrm{l}$; range 